Supporting Information for

\title{
Mechanical Properties of a Library of Low-Bandgap Polymers
}

Bérenger Roth ${ }^{\mathrm{a}}{ }^{\dagger}$ Suchol Savagatrup ${ }^{\mathrm{b}}{ }^{\dagger}$ Nathaniel V. de los Santos ${ }^{\mathrm{b}}$, Ole Hagemann ${ }^{\mathrm{a}}$, Jon E. Carlé $^{\mathrm{a}}$, Martin Helgesen ${ }^{\mathrm{a}}$, Francesco Livi ${ }^{\mathrm{a}}$, Eva Bundgaard ${ }^{\mathrm{a},}$ Roar R. Søndergaard ${ }^{\mathrm{a}}$, Frederik C. $\mathrm{Krebs}^{\mathrm{a}}$, and Darren J. Lipomi ${ }^{\mathrm{b}, *}$

${ }^{a}$ Department of Energy Conversion and Storage, Technical University of Denmark, Frederiksborgvej 399, DK-4000 Roskilde, Denmark.

${ }^{b}$ Department of NanoEngineering, University of California

San Diego, 9500 Gilman Drive, Mail Code 0448, La Jolla, CA 92093-0448, USA

*Author to whom correspondence should be addressed: dlipomi@ucsd.edu $\left({ }^{\dagger}\right.$ Equal contribution) 


\section{Film thicknesses for the calculation of tensile moduli}

Table S1. Values of film thickness used in the calculation of tensile moduli reported in this work. The solutions for all polymers were made by dissolving $10 \mathrm{mg} \mathrm{mL}^{-1}$ in $\mathrm{CHCl}_{3}$. The spin speeds were set at 500, 1000, and 200 RPM. The variations in thicknesses among different polymer were the result of differing viscosities. The slopes and the $\mathrm{R}^{2}$ values of the linear fits are also given for each polymer.

\begin{tabular}{|c|c|c|c|c|c|c|c|c|}
\hline \multirow[b]{2}{*}{ Polymer } & \multicolumn{2}{|c|}{500 RPM } & \multicolumn{2}{|c|}{1000 RPM } & \multicolumn{2}{|c|}{2000 RPM } & \multirow[t]{2}{*}{$\begin{array}{l}\text { Slope of } \\
\text { linear fit }\end{array}$} & \multirow[t]{2}{*}{$\mathbf{R}^{2}$} \\
\hline & $\begin{array}{c}\text { Film } \\
\text { thickness } \\
(\mathrm{nm})\end{array}$ & $\begin{array}{c}\text { Buckling } \\
\text { wavelength } \\
\text { (um) }\end{array}$ & $\begin{array}{c}\text { Film } \\
\text { thickness } \\
(\mathrm{nm})\end{array}$ & $\begin{array}{c}\text { Buckling } \\
\text { wavelength } \\
\text { (um) }\end{array}$ & $\begin{array}{c}\text { Film } \\
\text { thickness } \\
(\mathrm{nm})\end{array}$ & $\begin{array}{c}\text { Buckling } \\
\text { wavelength } \\
\text { (um) }\end{array}$ & & \\
\hline A1D5 & 409 & 17.18 & 215 & 11.40 & 165 & 8.94 & 32.65 & 0.99 \\
\hline A1D9 & 289 & 11.17 & 215 & 8.75 & 178 & 6.78 & 38.37 & 0.98 \\
\hline A2D1 & 231 & 15.78 & 210 & 12.41 & 153 & 8.93 & 81.65 & 0.96 \\
\hline A2D2 & - & - & - & - & - & - & - & - \\
\hline A2D3 & 262 & 10.76 & 209 & 7.28 & 169 & 5.49 & 57.38 & 0.99 \\
\hline A2D5 & 263 & 11.26 & 209 & 9.12 & 176 & 7.74 & 40.29 & 1.00 \\
\hline A2D9 & 299 & 9.40 & 242 & 6.95 & 174 & 4.97 & 35.46 & 0.99 \\
\hline A3D1 & 318 & 15.77 & 285 & 13.30 & 258 & 12.13 & 61.62 & 0.98 \\
\hline A3D3 & 130 & 8.06 & 108 & 6.69 & 93 & 5.09 & 78.50 & 0.98 \\
\hline A3D6 & 501 & 17.95 & 345 & 13.65 & 268 & 11.34 & 28.34 & 1.00 \\
\hline A3D7 & 129 & 5.08 & 98 & 3.87 & 80 & 3.28 & 36.83 & 1.00 \\
\hline A3D8 & 170 & 7.67 & 143 & 6.22 & 121 & 5.16 & 51.43 & 1.00 \\
\hline A3D9 & - & - & - & - & - & - & - & - \\
\hline A4D2 & 216 & 11.86 & 197 & 9.83 & 145 & 7.13 & 63.65 & 0.97 \\
\hline A4D5 & 300 & 14.75 & 214 & 9.92 & 178 & 8.04 & 55.50 & 1.00 \\
\hline A4D9 & 227 & 9.44 & 156 & 7.12 & 136 & 5.81 & 38.28 & 0.98 \\
\hline A5D1 & 233 & 11.50 & 166 & 7.37 & 129 & 5.46 & 58.72 & 1.00 \\
\hline A5D2 & 88 & 5.02 & 77 & 4.24 & 47 & 2.86 & 51.51 & 0.99 \\
\hline A5D6 & 354 & 20.26 & 303 & 15.52 & 222 & 12.20 & 59.15 & 0.95 \\
\hline A5D7 & 511 & 16.07 & 392 & 12.97 & 306 & 9.98 & 29.46 & 0.99 \\
\hline A5D9 & 538 & 18.17 & 397 & 12.66 & 342 & 9.85 & 41.73 & 1.00 \\
\hline A6D1 & 264 & 9.92 & 194 & 7.48 & 162 & 6.28 & 35.42 & 1.00 \\
\hline A6D2 & 334 & 15.66 & 271 & 11.07 & 215 & 8.23 & 62.84 & 0.99 \\
\hline A6D9 & 254 & 10.02 & 192 & 7.81 & 141 & 6.54 & 30.90 & 0.99 \\
\hline A7D7 & 194 & 9.79 & 133 & 7.71 & 113 & 6.57 & 38.46 & 0.99 \\
\hline A8D1 & 239 & 17.24 & 217 & 14.56 & 154 & 8.65 & 99.26 & 1.00 \\
\hline A8D2 & 229 & 14.67 & 211 & 12.69 & 154 & 9.46 & 65.96 & 0.98 \\
\hline A8D3 & 226 & 13.92 & 174 & 9.52 & 134 & 7.39 & 71.68 & 0.99 \\
\hline A8D5 & 254 & 10.68 & 221 & 8.61 & 179 & 6.66 & 52.95 & 0.99 \\
\hline A8D7 & 246 & 10.16 & 216 & 8.65 & 148 & 6.15 & 40.06 & 0.99 \\
\hline A8D8 & - & - & - & - & - & - & - & - \\
\hline A9D1 & 367 & 12.61 & 272 & 8.19 & 220 & 6.61 & 41.58 & 0.99 \\
\hline A9D7 & 259 & 9.83 & 205 & 7.15 & 170 & 6.09 & 42.64 & 0.98 \\
\hline
\end{tabular}




\begin{tabular}{l|cc|cc|cc|cc}
\hline & \multicolumn{2}{|c|}{$\mathbf{5 0 0}$ RPM } & \multicolumn{2}{|c|}{$\mathbf{1 0 0 0}$ RPM } & \multicolumn{2}{|c|}{ 2000 RPM } & $\begin{array}{c}\text { Slope of } \\
\text { linear fit }\end{array}$ & $\mathbf{R}^{\mathbf{2}}$ \\
\hline Polymer & $\begin{array}{c}\text { Film } \\
\text { thickness } \\
(\mathrm{nm})\end{array}$ & $\begin{array}{c}\text { Buckling } \\
\text { wavelength } \\
\text { (um) }\end{array}$ & $\begin{array}{c}\text { Film } \\
\text { thickness } \\
(\mathrm{nm})\end{array}$ & $\begin{array}{c}\text { Buckling } \\
\text { wavelength } \\
\text { (um) }\end{array}$ & $\begin{array}{c}\text { Film } \\
\text { thickness } \\
(\mathrm{nm})\end{array}$ & $\begin{array}{c}\text { Buckling } \\
\text { wavelength } \\
\text { (um) }\end{array}$ & & \\
\hline A10D1 & - & - & - & - & - & - & - & - \\
A10D2 & - & - & - & - & - & - & - & - \\
A10D3 & - & - & - & - & - & - & - & - \\
A10D6 & - & - & - & - & - & - & - & - \\
A10D7 & 392 & 11.10 & 309 & 8.31 & 250 & 6.70 & 31.14 & 1.00 \\
A10D8 & 265 & 8.43 & 241 & 6.86 & 180 & 4.96 & 38.73 & 0.96 \\
A10D9 & - & - & - & - & - & - & - & - \\
\hline A12D5 & 115 & 6.36 & 100 & 5.47 & 79 & 4.76 & 44.24 & 0.98 \\
A12D7 & 212 & 8.31 & 185 & 6.62 & 149 & 5.24 & 47.77 & 0.98 \\
A12D9 & 141 & 6.15 & 110 & 5.15 & 84 & 4.20 & 33.99 & 1.00 \\
\hline A13D1 & 238 & 9.81 & 146 & 6.27 & 119 & 4.27 & 44.62 & 0.98 \\
A13D7 & 331 & 11.82 & 289 & 10.09 & 256 & 9.12 & 36.04 & 0.99 \\
A13D9 & 211 & 7.24 & 184 & 6.44 & 170 & 5.86 & 33.41 & 0.99 \\
\hline A14D1 & 154 & 6.83 & 104 & 5.38 & 92 & 4.51 & 34.72 & 0.96 \\
A14D2 & 186 & 7.99 & 156 & 6.69 & 116 & 5.31 & 38.34 & 1.00 \\
A14D3 & 116 & 4.72 & 88 & 3.70 & 71 & 2.82 & 40.80 & 0.99 \\
A14D7 & 165 & 9.05 & 139 & 7.61 & 88 & 5.32 & 48.11 & 1.00 \\
A14D9 & 322 & 9.53 & 288 & 7.84 & 222 & 6.42 & 29.78 & 0.95 \\
\hline
\end{tabular}

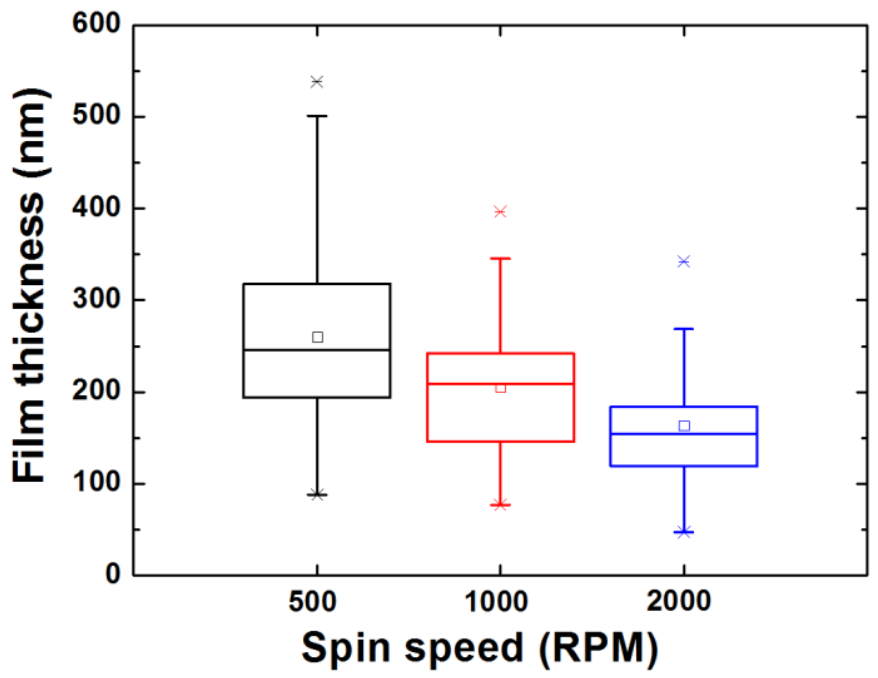

Figure S1. Box diagram of the film thickness used in the measurement of the tensile moduli separated by the spin speed. 


\section{Film thickness for crack-onset strain measurements}

Table S2. Averaged film thicknesses and standard deviations of all the polymer films for the measurement of crack-onset strain.

\begin{tabular}{|c|c|c|c|c|c|}
\hline Polymer & $\begin{array}{c}\text { Averaged } \\
\text { thickness }(\mathrm{nm})\end{array}$ & $\begin{array}{c}\text { Standard } \\
\text { Deviation }(\mathrm{nm})\end{array}$ & Polymer & $\begin{array}{c}\text { Averaged } \\
\text { thickness (nm) }\end{array}$ & $\begin{array}{c}\text { Standard } \\
\text { Deviation }(\mathrm{nm})\end{array}$ \\
\hline A1D5 & 117.3 & 3.5 & A8D1 & 374.3 & 14.6 \\
\hline A1D9 & 358.7 & 21.5 & A8D2 & 143.8 & 1.5 \\
\hline A2D1 & 168.4 & 21.0 & A8D3 & 160.3 & 23.0 \\
\hline A2D2 & 190.0 & 9.7 & A8D5 & 290.5 & 29.0 \\
\hline A2D3 & 394.6 & 4.3 & A8D7 & 209.7 & 12.6 \\
\hline A2D5 & 148.9 & 2.8 & A8D8 & 266.7 & 15.0 \\
\hline A2D9 & 317.9 & 3.8 & A9D1 & 242.8 & 8.0 \\
\hline A3D1 & 96.8 & 2.1 & A9D7 & 310.1 & 26.0 \\
\hline A3D3 & 368.5 & 32.5 & A10D1 & 186.4 & 17.3 \\
\hline A3D6 & - & - & A10D2 & 107.9 & 0.8 \\
\hline A3D7 & 206.0 & 5.6 & A10D3 & 179.9 & 3.9 \\
\hline A3D8 & - & - & A10D6 & 152.5 & 4.3 \\
\hline A3D9 & 150.7 & 2.5 & A10D7 & 315.6 & 6.4 \\
\hline A4D2 & 316.1 & 6.3 & A10D8 & 221.8 & 13.5 \\
\hline A4D5 & 111.8 & 4.1 & A10D9 & 280.2 & 11.2 \\
\hline A4D9 & 137.2 & 9.2 & A12D5 & 274.5 & 10.5 \\
\hline A5D1 & 163.4 & 3.1 & A12D7 & 249.1 & 40.0 \\
\hline A5D2 & - & - & A12D9 & 268.7 & 37.5 \\
\hline A5D6 & 236.2 & 19.0 & A13D1 & 109.7 & 2.6 \\
\hline A5D7 & 138.9 & 5.3 & A13D7 & 226.7 & 5.3 \\
\hline A5D9 & 260.6 & 15.8 & A13D9 & 331.3 & 22.6 \\
\hline A6D1 & 185.1 & 7.4 & A14D1 & 271.4 & 6.0 \\
\hline A6D2 & 327.7 & 31.0 & A14D2 & 216.8 & 5.1 \\
\hline A6D9 & 165.2 & 8.0 & A14D3 & 147.4 & 0.7 \\
\hline \multirow{2}{*}{ A7D7 } & \multirow{2}{*}{167.9} & \multirow{2}{*}{5.0} & A14D7 & 198.3 & 6.5 \\
\hline & & & A14D9 & - & - \\
\hline
\end{tabular}



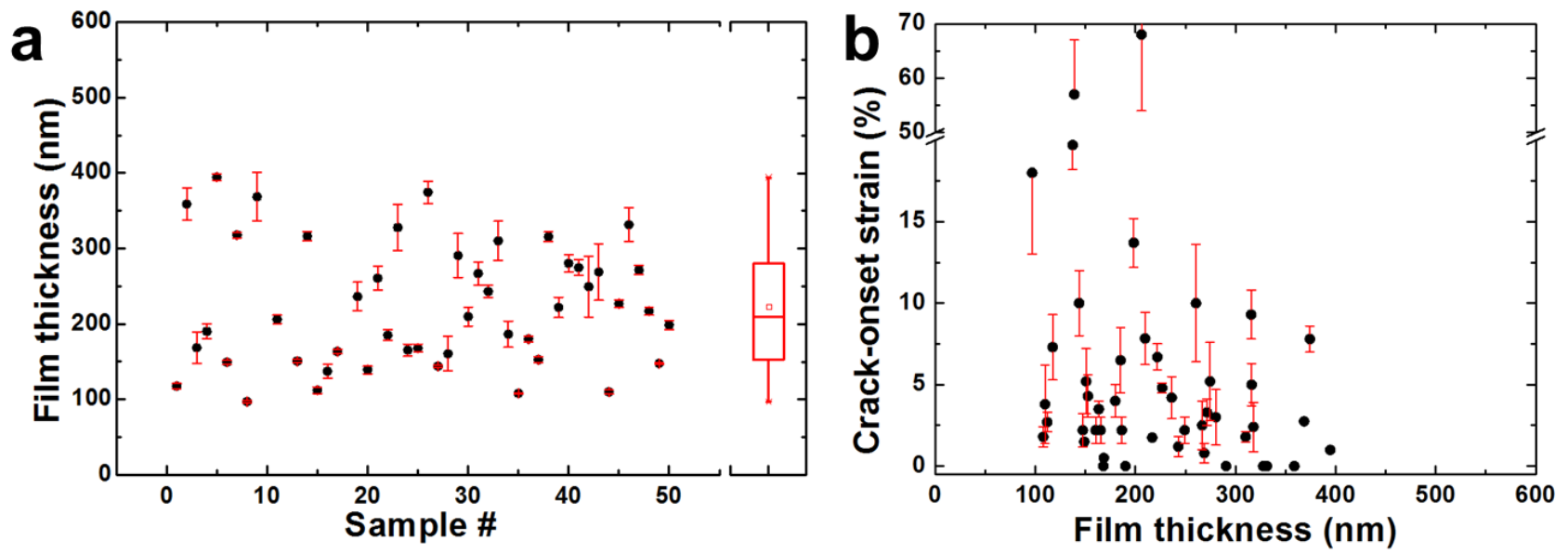

Figure S2. (a) Film thicknesses for all the polymer samples used in the measurement of the crack-onset strains. (b) Crack-onset strain as a function of film thickness. We found no correlation between the crackonset and the film thickness for this range of thicknesses. 